\title{
Paraganglioma of the mediastinum: challenges in diagnosis and surgical management
}

\author{
Ori Wald, Oz M Shapira, Aiman Murar and Uzi Izhar*
}

\begin{abstract}
Mediastinal paraganglioms are rare, highly vascularized tumors arising from chromaffin tissue located in the para-aortic ganglia. Tumors tend to invade bordering structures and may also form metastasis. Up to 50\% of patients are asymptomatic and diagnosis is incidental. Presenting symptoms are related to catecholamine hypersecretion or to a mass effect. Complete surgical resection remains the standard of care due to malignant potential of the tumor and poor response to chemotherapy or radiation. Strategic location of the tumor in proximity to great vessels, trachea, and recurrent laryngeal nerve poses challenge for the surgeon. We report a case of a 59-year old asymptomatic female who was incidentally diagnosed with a middle mediastinal mass on a positron-emission tomography (PET-CT) scan performed as part of breast cancer surveillance. Complete resection of the tumor was achieved using cardiopulmonary bypass. The patient recovered uneventfully and in a ten-month follow up there is no evidence of recurrence.
\end{abstract}

\section{Introduction}

Ninety percent of chromaffin-cell-originating tumors are located in the adrenal gland and termed pheochromocytomas. The remaining ten percent are extra adrenal and are termed paragangliomas. Paragangliomas appear in the abdomen, pelvis, neck and mediastimun. Mediastinal paraganglioma originate from para-aortic (middle mediatsinum) and para-vertebral (posterior mediatsinum) sympathetic chain ganglia $[1,2]$. Similar to pheochromocytoma, paraganglioma tumors may secrete catecholamines, however in majority of cases they are nonfunctional. Up to $50 \%$ of patients are asymptomatic and the diagnosis is incidental [1]. Clinical symptoms may be related to catecholamine hypersecretion (hypertention/ hyperhydrosis) or to a mass effect resulting in complains of hoarseness, dysphagia, shortness of breath and chest pain [3].

\section{Case}

A 54-year-old woman was referred for resection of an asymptomatic middle mediastinal mass. Her past medical history was remarkable for right breast carcinoma treated with lumpectomy, axillary lymph node dissection, adjuvant chemotherapy and irradiation sixteen years prior to

\footnotetext{
* Correspondence: IZHARU@hadassah.org.il

1 Department of Cardiothoracic Surgery, Hadassah University Hospital, Jerusalem, 91120 P.O.B 12000, Israel

Full list of author information is available at the end of the article
}

this admission. Six years later a right para-vertebral desmoid tumor was completely resected. One year ago a second right breast carcinoma was diagnosed and completion mastectomy was performed. A positron emission tomography - computerized tomography (PETCT scan) performed prior to her admission as part of her oncological follow-up revealed a 5 - $\mathrm{cm}$ middle mediastinal mass with a standardized uptake value (SUV) of 20 (Fig 1A). With a differential diagnosis of an infectious process a course of antibiotics was administered without response. Repeat computerized tomography of the chest (CT scan) demonstrated a 5-cm mass, located between the aorta and the superior vena cava, compressing the right pulmonary artery, and adherent to the anterior tracheal wall (Fig 1B, 1C). Trans-bronchial biopsy was suggestive of a typical carcinoid tumor and the patient was referred to surgery.

Upon admission, the patient denied any symptoms related neither to catecholamine hyper-secretion nor to carcinoid syndrome. Physical examination and routine laboratory results were unremarkable.

The operation was performed via a median sternotomy. A soft, highly vascularized, $1.5 \times 2.5 \times 5.5 \mathrm{~cm}$ mass located between the aorta, the superior vena cava and the right atrium was identified. The tumor compressed the right pulmonary artery, and was densely adherent to the aorta and the anterior wall of the trachea with a very rich network of small blood vessels (Fig 2). Frozen section 

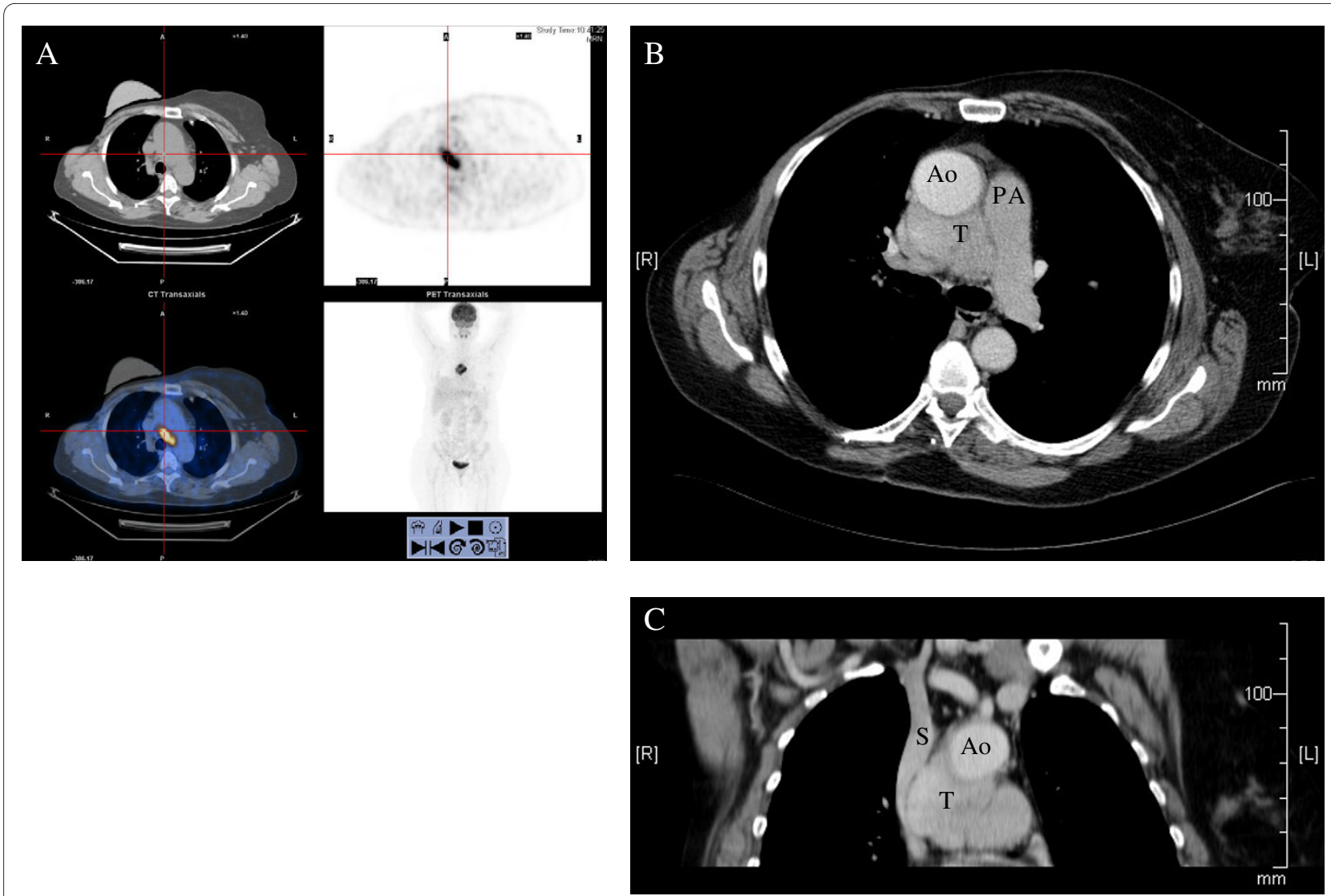

Figure 1 A - PET CT scan image showing a middle mediastinal mass of $5 \mathbf{c m}$ in diameter with an SUV of 20. B, C - Axial and coronal CT scan images showing the tumor ( $\mathrm{T}$ ) adjacent to the aorta ( $\mathrm{Ao}$ ) and pulmonary artery (PA). The compressed superior vena cava is seen in coronal view (SVC).

confirmed the diagnosis of a neuroendocrine tumor with a low mitotic index. Because of these findings we elected to remove the tumor using cardiopulmonary bypass to allow complete and safe resection. We performed a right femoral artery and the right atrium cannulaion, allowing manipulation and possible excision of the ascending aorta. The mass was completely resected. Postoperative course was remarkable for a transient left vocal cord paralysis. Final pathological examination demonstrated the characteristics architecture of paraganglioma. The tumor cells stained positive for synaptophysin chromogranin and sustentacular cells stained positive to S-100. Proliferation index was 5\%. Cytology result of pericardial effusion did not show malignant cells.

The patient has been followed-up since surgery. Currently she is asymptomatic. Both chest MRI and a whole body FDG PET-CT scan, performed 10 months postoperative, did not show evidence of recurrence.

\section{Comment}

The diagnosis of a paraganglioma is based on clinical symptoms, imaging tests and urinary essays of catecholamine metabolites. Patients with inactive tumors may present with large tumors that compress or invade neighboring structures $[1,4]$.

Paragangliomas have typical imaging characteristics on $\mathrm{CT}$ and magnetic resonance imaging (MRI) scans. Paragnagliomas are usually located in the bifurcation of great vessels and show intense and homogeneous enhancement except for necrotic areas that enhanced poorly. MRI images show intermediate signal intensity on T1weighted images and high signal intensity on T2weighted images [2]. ${ }^{123}$ I-MIBG (123I-metaiodobenzylguanidine) scintigraphy and PET-CT scan with 18-FDG (18-fluorodeoxyglucose) are used for localization and staging of these tumors when appropriate. CT-guided needle biopsy is not mandatory and, in fact, can be hazardous due to the proximity of the tumor to the great vessels and its intense vascularity. Our patient's history suggested a differential diagnosis for the current mass of either tumor recurrence or a third primary tumor. Therefore preoperative tissue diagnosis was indicated. Needlebiopsy-based tissue diagnosis of paraganglioma is difficult and complete pathological examination including tumor morphology and structure and specific stains are necessary to achieve accurate diagnosis. 


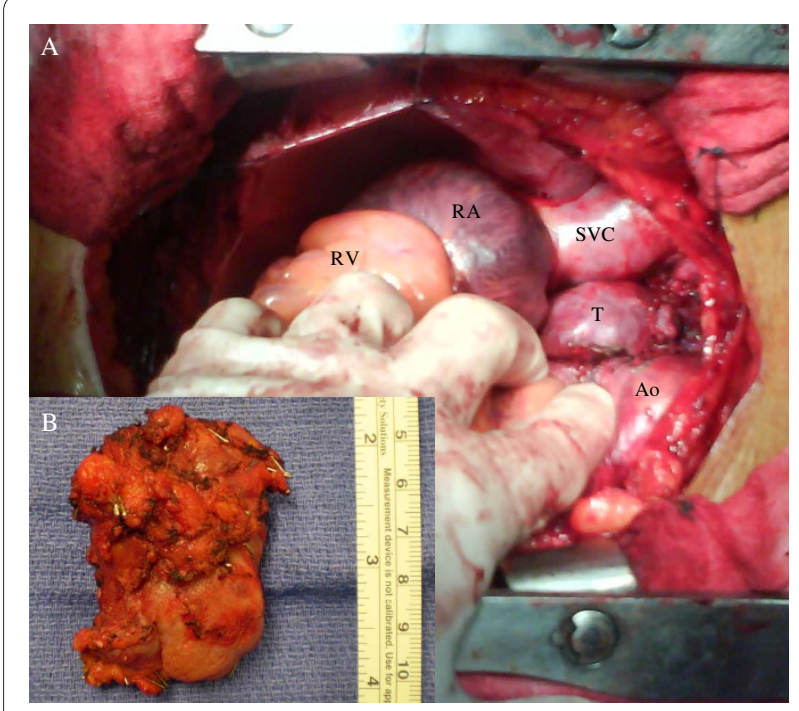

Figure 2 A - Intraoperative view: the tumor $(\mathrm{T})$ is seen between the aorta (Ao) and superior vena cava (S). Right atrium (RA) Right ventricle (RV). B - resected tumor is shown.

Complete resection is the standard of care of paraganglioma, affording the patient with the best chance of cure since these tumors are relatively resistant to chemotherapy and irradiation [5]. The highly vascular nature of these tumors and strategic anatomical locations make complete resection demanding, and often mandates the use of median sternotomy and cardiopulmonary bypass [6,7]. In most cases these tumors can be removed in a single-stage operation. A recent report described a twostage approach for resection of a paraganglioma invading the pulmonary artery and ascending aorta [8].

The two major concerns involving resection of mediastinal paragngliomas include intraoperative bleeding and catecholamine crises in patients with metabolically active tumors. The highly vascular nature of the tumor, the proximity and invasion to the great arteries and the systemic anticoagulation necessary for cardiopulmonary bypass - all contribute to a high risk of bleeding. [9]. Hormonal-related crises are uncommon but are associated with significant morbidity and mortality $[1,10]$. Meticulous surgical technique and tight preoperative blood pressure control are the key steps in prevention and management of these complication [7].

Prognosis after complete resection is favorable. Lamy et al reported a follow up of 79 patients with middle mediastinal paragangliomas over a period of 180 months. Among these patients overall survival was $62.0 \%$, mean survival time was $98.2+/$ - 11.7 months (mean $+/$ - standard error). For patient undergoing complete resection survival was $84.6 \%$, mean survival time was $125.7+/-18.7$ months (mean $+/$ - standard error). For patient undergoing incomplete resection survival was $50.0 \%$ mean sur- vival time was $71.5+/-13.8$ months (mean $+/$ - standard error) [5]. In a literature review of extraadrenal chromaffin cells tumors Erickson et al has reported a surgical cure rate of $69 \%$ [10].

In summary, paragnagliomas should be included in the differential diagnosis of a middle mediastinal mass. Complete surgical resection remains the standard of care and is associated with excellent survival. Life-long surveillance for local recurrence and metastatic spread is mandatory.

\section{Competing interests \\ The authors declare that they have no competing interests.}

\section{Authors' contributions}

OW reviewed the literature and wrote the manuscript, UI and OMS operated on the patient and edited the manuscript. IM took part in operation, and prepared the figures. All authors read and approved the final manuscript.

\section{Author Details}

Department of Cardiothoracic Surgery, Hadassah University Hospital, Jerusalem, 91120 P.O.B 12000, Israel

Received: 21 November 2009 Accepted: 31 March 2010 Published: 31 March 2010

\section{References}

1. Young WF Jr: Paragangliomas: clinical overview. Ann N Y Acad Sci 2006, 1073:21-29.

2. Balcombe J, Torigian DA, Kim W, Miller WT Jr: Cross-sectional imaging of paragangliomas of the aortic body and other thoracic branchiomeric paraganglia. AJR Am J Roentgenol 2007, 188:1054-1058.

3. Brown ML, Zayas GE, Abel MD, Young WF Jr, Schaff HV: Mediastinal paragangliomas: the mayo clinic experience. Ann Thorac Surg 2008, 86:946-951.

4. Ramos R, Moya J, Villalonga R, Morera R, Ferrer G: Mediastinal aortosympathetic paraganglioma: report of two cases. Asian Cardiovasc Thorac Ann 2007, 15:e49-51.

5. Lamy AL, Fradet GJ, Luoma A, Nelems B: Anterior and middle mediastinum paraganglioma: complete resection is the treatment of choice. Ann Thorac Surg 1994, 57:249-252.

6. Andrade CF, Camargo SM, Zanchet M, Felicetti JC, Cardoso PF: Nonfunctioning paraganglioma of the aortopulmonary window. Ann Thorac Surg 2003, 75:1950-1951.

7. Paul S, Jain SH, Gallegos RP, Aranki SF, Bueno R: Functional paraganglioma of the middle mediastinum. Ann Thorac Surg 2007 83:e14-16.

8. Qedra N, Kadry M, Buz S, Meyer R, Ewert P, Hetzer R: Aorticopulmonary paraganglioma with severe obstruction of the pulmonary artery: successful combined treatment by stenting and surgery. Ann Thorac Surg 2009, 87:1284-1286.

9. Otake Y, Aoki M, Imamura N, Ishikawa M, Hashimoto K, Fujiyama R: Aortico-pulmonary paraganglioma: case report and Japanese review. Jpn J Thorac Cardiovasc Surg 2006, 54:212-216.

10. Erickson D, Kudva YC, Ebersold MJ, Thompson GB, Grant CS, van Heerden JA, Young WF Jr: Benign paragangliomas: clinical presentation and treatment outcomes in 236 patients. J Clin Endocrinol Metab 2001, $86: 5210-5216$

doi: 10.1186/1749-8090-5-19

Cite this article as: Wald et al., Paraganglioma of the mediastinum: challenges in diagnosis and surgical management Journal of Cardiothoracic Surgery 2010, 5:19 